\title{
Structured latent growth curves for twin data
}

\author{
Michael C Neal $e^{1}$ and John J McArdle \\ ${ }^{1}$ Department of Psychiatry, Medical College of Virginia, Richmond \\ ${ }^{2}$ Department of Psychology, Gilmer Hall, University of Virginia, Charlottesville, VA, USA
}

\begin{abstract}
We describe methods to fit structured latent growth curves to data from $M Z$ and $D Z$ twins. The well-known Gompertz, logistic and exponential curves may be written as a function of three components - asymptote, initial value, and rate of change. These components are allowed to vary and covary within individuals in a structured latent growth model. Such models are highly economical, requiring a small number of parameters to describe covariation across many occasions of measurement. We extend these methods to analyse longitudinal data from MZ and DZ twins and focus on the estimation of genetic and environmental variation and covariation in each of the asymptote, initial and rate of growth factors. For illustration, the models are fitted to longitudinal Bayley Infant Mental Development Scale data published by McArdle (1986). In these data, all three components of growth appear strongly familial with the majority of variance associated with the shared environment; differences between the models were not great. Occasionspecific residual factors not associated with the curve components account for approximately $40 \%$ of variance of which a significant proportion is additive genetic. Though the growth curve model fit less well than some others, they make restrictive, falsifiable predictions about the mean, variance and twin covariance of other (not yet measured) occasions of measurement. Twin Research (2000) 3, 165-177.
\end{abstract}

Keywords: growth curves, genes, dynamical models, twins, cognition, development, Bayley Infant Mental Development, methodology

\section{Introduction}

Growth and decay are essential properties of living organisms. An improved understanding of the mechanisms of development seems fundamental to explaining individual differences in almost all biological science, and behavior genetics is no exception. In this paper, we describe, extend and apply growth curve models which predict changes in mean, variance, and covariance over time. These curve models can predict non-monotonic increases and decreases in means and covariances, and the two types of statistics do not necessarily change in parallel.

Growth curve models are well-suited to data where the number of occasions of measurement is large, because they describe changes in mean, variance and covariance with a limited number of parameters. We begin with a review of the conceptual background to the curves, and then show how they may be used to derive an appropriate set of factor loadings for model-fitting. Our methods are based on those previously published by Browne ${ }^{1}$ and Browne and Du Toit. ${ }^{2}$ We extend them to model genetic and environmental components of variation

Correspondence: Michael C Neale, Department of Psychiatry, Medical College of Virginia, Box 080710, Richmond, VA 23298, USA. E-mail: neal e@psycho.psi.vcu.edu

Received 13 January 2000; accepted 18 February 2000 in growth curves, using data from relatives, and provide $M x$ scripts to make the methods readily available.

At present, behavior genetic analyses of longitudinal data usual ly employ one of a few basic models: a) the Cholesky factorization; b) a Markov chain; or c) growth curves. As described by Neale and Cardon, ${ }^{3}$ the Chol esky factor model, also known as the square root factorization, ${ }^{4,5}$ (see Figurela) is a transformation of a covariance matrix. This model, presented as a path diagram in Figure la, has the same number of parameters as there are covariances $(m(m+1) / 2$ for $\mathrm{m}$ variables), and will always fit perfectly. In a genetic Cholesky model, the additive genetic (A), common (C) or specific (E) environmental covariance matrices are each decomposed into their Cholesky factors. While they describe the covariance of each of the components perfectly, the Cholesky factor model may fail because the within-person covariances are estimated from four different sources in the classical twin model, and these replicate statistics may be unstable. Similarly, the cross-twin cross-variable covariances may be non-symmetric and therefore contribute to lack of fit of the Chol esky. Such statistical fluctuations of the data do not reflect the appropriateness of the Cholesky as a model. Other causes of failure - such as DZ covariances being less than half the corresponding MZ covariances, or phenotypic variance differences between 
$M Z$ and DZ twins - would be informative about the general suitability of the ACE model for the data, but still do not test the number of factors or the triangular pattern of their loadings.

In contrast to its use in the mul tivariate analysis of a set of variables, the Cholesky decomposition may have a useful conceptual interpretation when applied to longitudinal data which are ordered from timel to time $\mathrm{m}$. All factors are constrained to impact variation at current and later - but not earlier - occasions. Thus the first factor (F1) may create variation at all occasions, but F2 may influence all occasions except the first. Just as the model may predict any change in variation and covariation over time, so it can predict any pattern of change in means. However, this explanatory power can be seen as a disadvantage, because the model is not falsifiable. No pattern of genetic covariances over time exists that could not be accounted for by the Chol esky model. Even worse, it makes no prediction about genetic variation or covariation on future, not yet measured, occasions.
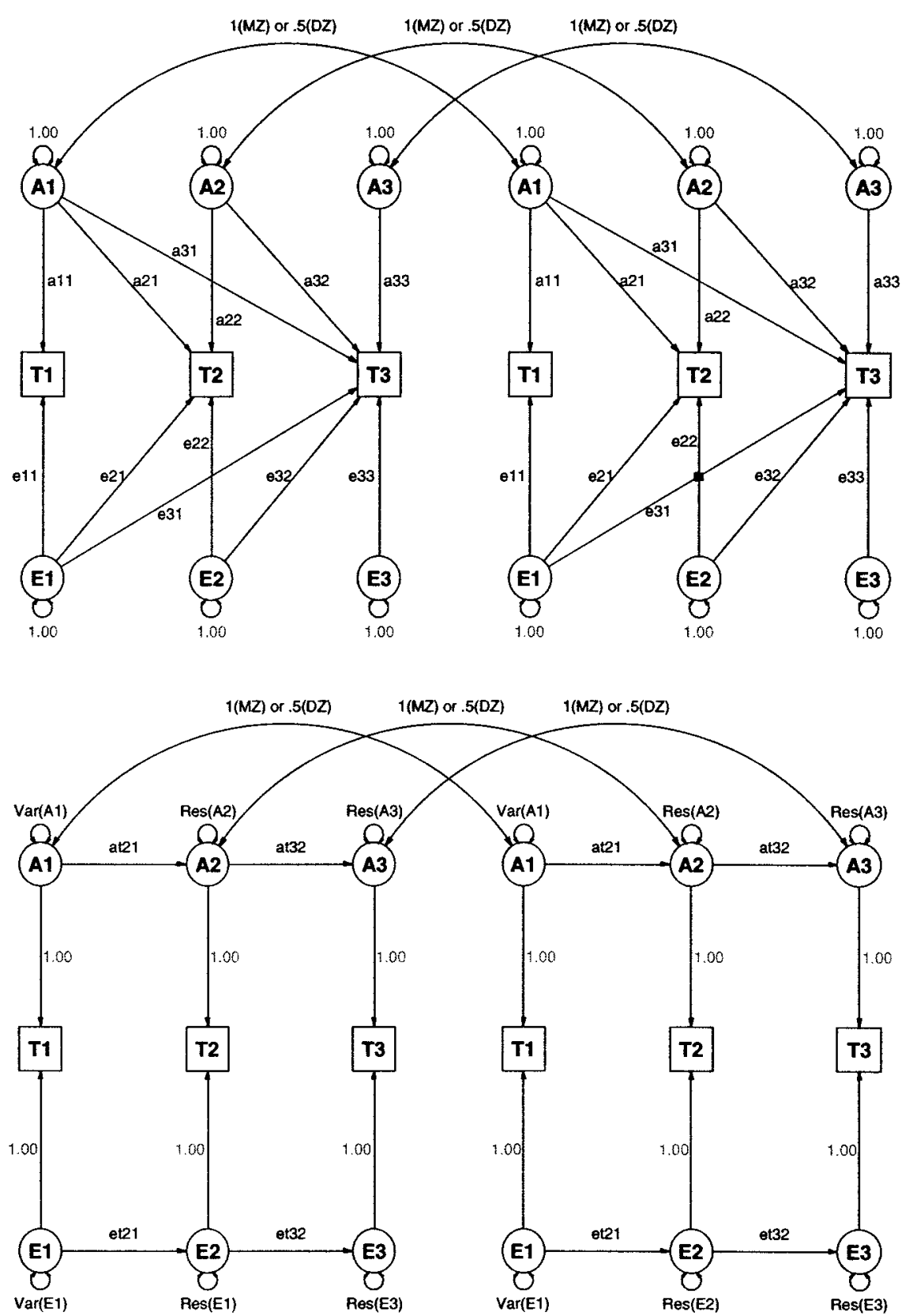

Figure 1 Two path models for longitudinal data from an individual: (top) Cholesky factor; and (bottom) Simplex or Markov 
The 'Simplex' or 'Markov chain' model of Figure $1 \mathrm{~b}$ provides an al ternative account of changes in variance over time. Dolan and colleagues ${ }^{6,7}$ describe a constrained version of this model which specifies a linear relationship between longitudinal changes in variance and concurrent changes in mean. In contrast to the Cholesky factor model, the simplex model makes highly restrictive predictions about covariances and hence is falsifiable with sufficient occasions. ${ }^{8}$ Differences in mean and variance (either at the phenotypic or genetic factor level) may be rel ated in a non-linear fashion, and the linear model may fail. For example, a decrease in variance might be accompanied by an increase in mean on one occasion, while at other times both may increase or decrease. Certain growth curve models predict more complex patterns of relationship between mean, variance and covariance, and they may be empirically tested against simpler models; hence our interest in them.

A popular, modern approach to the study and modeling of change is to use dynamical systems theory. ${ }^{1,9}$ Essentially these methods focus on the rate of change of a variable of interest (ie its slope or partial derivative) as a way to predict the level at a series of points in time. These methods have many applications, including pollution levels, disease epidemics, supermarket queues, population growth, radioactive decay, weather and chaotic systems. In this paper we relate structural equation modeling of genetically informative longi tudinal data to dynamical systems.

A growth curve approach to behavioral genetic analysis was introduced by Vandenberg and Falkner $^{10}$ who first fitted polynomial growth curves for each subject and then estimated heritabilities of the components. The latent variable growth model version of this idea was later presented by McArdle. ${ }^{11}$ Here we extend models of this type by incorporating structured factor loadings that represent el ementary theories about growth and change. In every case, we model the genetic and environmental sources of variance covariance in the latent growth components. This approach is analogous to using the phenotypic factors (common pathway) model rather than the biometric factors (independent pathway) model. 3,12 The latter framework, though appealing, cannot be identified for growth curves because there is no predicted mean difference between $M Z$ and $D Z$ twins. In this respect, the growth curve models are quite different from the Simplex and Cholesky which are variants of biometric factor models.

\section{Growth curves}

First we present the theoretical background behind three growth curves which display asymptotic behavior: the exponential, the logistic, and the gompertz. The basic idea behind these curves is that individual s start at some initial point, then grow at a rate which accelerates. This exponential growth characterizes the early stages of development, where the gradient gels steeper and steeper. However, it al so seems reasonable to assume that growth does not continue to accelerate forever; some limiting factor takes over, progressively slowing down growth, making the gradi ent less and less steep until the growth curve asymptotes at an equilibrium point. Different hypotheses about the type of growth and limiting factors lead to the different growth curves, and some of these will be discussed below.

\section{The exponential curve}

One of the simplest models for changing growth rate is where current growth rate is proportional to current size (see Malthus (1798) as reported by Murray ${ }^{13}$ ). If we were to plot size against time, the gradient would get steeper and steeper (see Figure2). Mathematically, we can write this concept as

$$
\frac{d y}{d t}=y(t) c \text {, }
$$

where $c$ is a constant and $y(t)$ is the size at time $t$. This differential equation involves both $y$ and the derivative of $y$ over time $t$. Some differential equations, including this one, can be solved to find an expression for $\mathrm{y}(\mathrm{t})$ by itself:

$$
y(t)=y(0) e^{t c}
$$

Braun $^{9}$ gives a good account of methods for solving such equations, and symbolic cal cul us software such as Mathematica ${ }^{14}$ may be used to verify results. Note how $y(t)$ increases exponentially over time from the

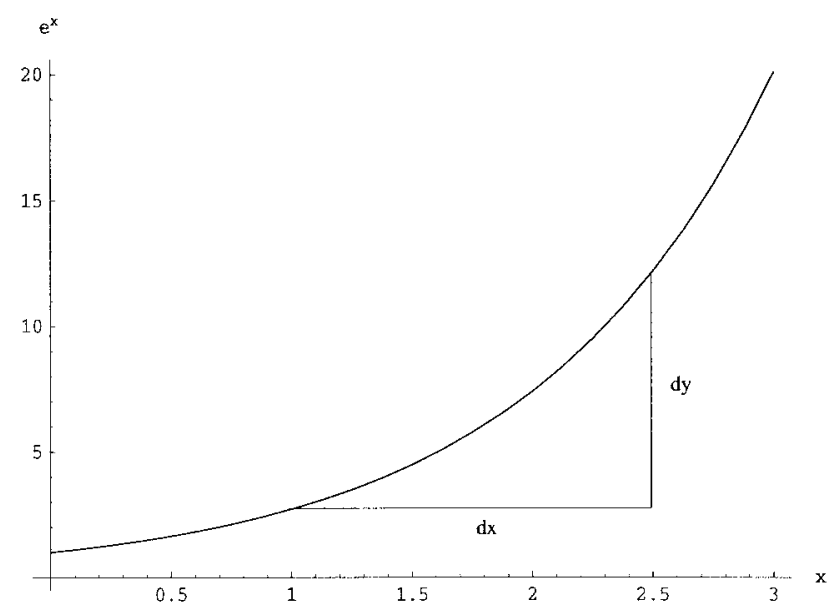

Figure 2 Plot of $y=e^{x}$ illustrating perpetual acceleration of growth for a system where growth rate is proportional to current size, dy and dx illustrate the derivative of the curve $d y / d x$ 
initial period $y(0)$ at time zero. While such growth may be a good approximation during initial stages of many biol ogical systems, it rarely applies to the later ones, so we need to add a 'carrying capacity' to represent an upper limit on size.

One form of asymptomatic behavior arises when the growth rate is proportional to the distance from the maximum height or asymptote $(a=y(\infty))$. In this case the differential may be written:

$$
\frac{d y}{d t}=c(a-y(t))
$$

which may solved to give

$$
y(t)=a-(a-y(0)) e^{-t c} .
$$

Note how this result scales the growth curve to lie between the initial value, $y(0)$, and the asymptote, a. When $t$ is zero, the exponential part gives $e^{0}=1$ so that $a-(a-y(0))=y(0)$. Conversely, when $t$ is large, $\mathrm{e}^{-\infty}=0$, giving $\mathrm{y}(\infty)=\mathrm{a}$. This meets the requirement for asymptotic behavior, as illustrated by the solid line in Figure 3.

\section{The logistic curve}

Another model for limited growth was proposed by Verhulst in $1838,{ }^{15}$ for changes in population size. He suggested that populations would grow in the exponential fashion of equation 1 above, but would be limited by the square of their size. Thus,

$$
\begin{aligned}
\frac{d y}{d t} & =a y(t)-b y(t)^{2} \\
& =a y(t)(1-y(t) b)
\end{aligned}
$$

where $a$ is the ultimate size or asymptote, and $b$ is the initial level. The solution of this equation is

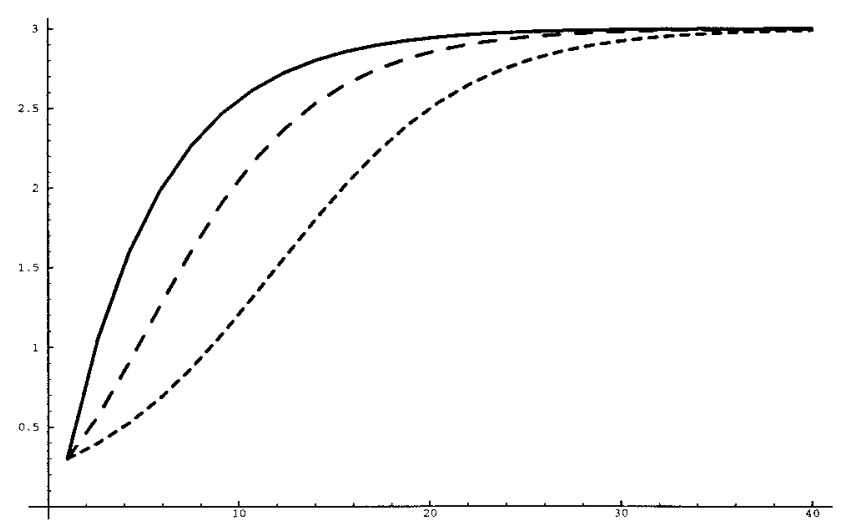

Figure 3 Growth curves from three functions that may display asymptotic behavior: exponential $(------)$, logistic $(---)$, Gompertz (- - - -). Parameter values used to draw the curves were: $a=3, i=0.3, r=0.2$

$$
y(t)=\frac{a y(0)}{b y(0)+(a-b y(0)) e^{-a t}}
$$

which is familiar to many researchers as the logistic curve. The middle line in Figure 3 is an example of a logistic curve. While it was developed for the growth of populations, it has (like many mathematical models) proved useful in a variety of contexts.

\section{The Gompertz curve}

A third model for asymptotic growth comes from Gompertz. ${ }^{16}$ It is similar to the logistic in that the rate of growth decays as a non-linear function of current height. Instead of the square of the current size, the product of logarithm of current height and the hei ght itself are used. We write the differential equation for this model as

$$
\frac{d y}{d t}=a y(t)-b \log [y(t)] y(t),
$$

so growth is initially exponential (from ay(t)), but as time passes the term on the right may counteract it. This equation can be solved and rearranged to give

$$
y(t)=\exp \left[\frac{a-e^{b(c-t)}}{b}\right]
$$

The behavior of the Gompertz curve varies according to the parameters; for some values of $b$ the curve does not asymptote but increases exponentially. An example Gompertz curve is the bottom line in Figure3. While the model was developed for cell reproduction during ontogeny (the growth of chicken hearts), it has been widely used in tumor growth and models of population death rates. ${ }^{17,18}$

We emphasize that the three curves presented here are only a small sample from the set of possibilities. However, they provide a foundation for a bridge between dynamical systems theory widely used in mathematical biology and structural equation modeling of genetically informative data.

\section{Modeling phenotypic data}

Now that we have mathematical equations for growth curves, we need to find a way to generate appropriate predictions for population means, variances and covariances across time. Each curve has parameters which control the initial level, the growth rate, and the asymptote. Suppose that there are latent factors which represent individual differences in these components, and that observed scores at different time points are a linear combination of these components and some random error. This can be described as a factor model with three latent 
factors, as shown in Figure4. Algebraically, we can write

$$
\mathbf{p}=\mathbf{F f}+\mathbf{E e},
$$

where $\mathbf{p}, \mathbf{f}$ and $\mathbf{e}$ are respectively vectors of phenotypes, factors and residual errors, $\mathbf{F}$ is the (full) matrix of factor loadings, and $\mathbf{E}$ is the diagonal matrix of loadings on the residual components. The rules of path analysis ${ }^{19-22}$ or multivariate path analysis ${ }^{23}$ may be useful to derive predicted covariances from this model as

$$
\Sigma=\text { FRF' }^{\prime}+\mathrm{EE}^{\prime}
$$

where $\mathbf{R}$ is the covariance matrix of the latent factors f. Population variation in the latent factors is assumed to be symmetric, so the predicted mean for occasion t equals the height of growth curve at that time point. We can write this model using factor means,

$$
\mu_{\mathrm{t}}=\mathbf{F} \mu_{\mathrm{f}},
$$

where $\mu_{t}$ is the $n_{t} \times 1$ vector of means, $n_{t}$ is the number of timepoints, and $\mu_{\mathrm{f}}=(\mathrm{a}, \mathrm{i}, 0)^{\prime}$ is the $1 \times 3$ vector of factor means. As we later show, within a computer program such as $\mathrm{Mx}$, the expression for the growth curve (Equations 4, 7 and 9) can be specified directly for the predicted means.

Our next task is to find expressions for the elements of $\mathbf{F}$, the factor loadings, that generate predicted covariances according to the growth curve model in use. We follow Browne ${ }^{1}$ by using a first-

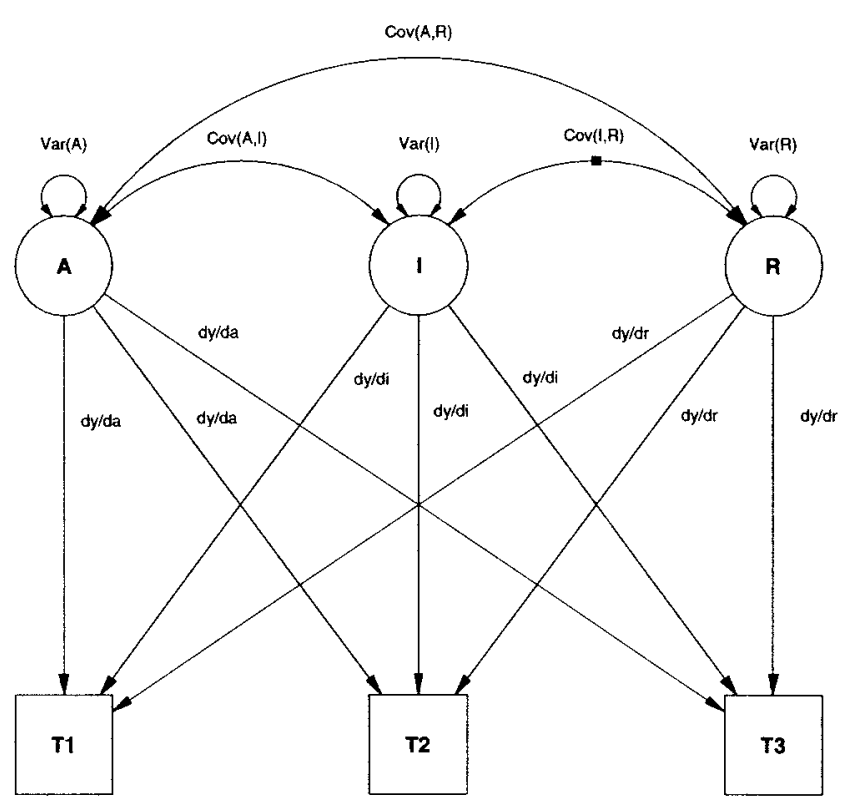

Figure 4 Three-factor model which represents variation in asymptote, initial level, and growth rate. All factors cause phenotypic variation on all occasions (T1, T2, T3), but the factor loadings are constrained according to the predictions of the model. Residual genetic and environmental components that influence only one time each are not shown order Taylor series to reproduce the growth curve. In brief, this involves use of the partial derivatives of the growth curve function (Equation 4 or 7 or 9 above) with respect to the parameters $a, b$ and $c$. For the vector of factor loadings from the asymptote factor under the exponential model we use the partial derivatives of Equation 4 with respect to a. These partial derivatives involve $t$, so the loadings are different from the different occasions $t=1,2 \ldots$. They form the columns of the matrix $\mathbf{F}$ in Equation 10. While these expressions have been published el sewhere, we reproduce them in Tablel for convenience. We could directly follow the equations for the three curves derived in equations 4,7 and 9 above, but a slight reparameterization due to Browne $^{1}$ makes the estimates easier to interpret, with a being the final asymptote and $\mathrm{i}$ being the value at time $t=0$ for all three curves.

Table 1 Exponential, logistic and Gompertz growth curve functions and their partial derivatives

\section{Exponential}

$$
F_{E}=a-(a-i) \exp [-(t-1) r]
$$

$\frac{d F_{E}}{d a}=1-\exp [-(t-1) r]$

$\frac{\mathrm{dF}_{\mathrm{E}}}{\mathrm{di}}=\exp [-(\mathrm{t}-1) \mathrm{r}]$

$\frac{d F_{E}}{d r}=(a-i)(t-1) \exp [-(t-1) r]$

Logistic

$$
F_{L}=\frac{a i}{i+(a-i) \exp [-(t-1) r]}
$$

$\frac{d F_{L}}{d a}=\frac{i-\exp [-(t-1) r] F_{L}}{i+(a-i) \exp [-(t-1) r]}$

$\frac{d F_{L}}{d i}=\frac{a-(1-\exp [-(t-1) r]) F_{L}}{i+(a-i) \exp [-(t-1) r]}$

$\frac{d F_{L}}{d r}=\frac{(a-i)(t-1) \exp [-(t-1) r] F_{L}}{i+(a-i) \exp [-(t-1) r]}$

Gompertz

$$
\begin{aligned}
F_{G} & =a \exp \left[\log \left[\frac{i}{a}\right] \exp [-(t-1) r]\right] \\
\frac{d_{F}}{d a} & =[1-\exp [-(t-1) r]] \exp \left[\log \left[\frac{i}{a}\right] \exp [-(t-1) r]\right]
\end{aligned}
$$

$\frac{d F G}{d i}=\frac{a}{i} \exp \left[-(t-1) r+\log \left[\frac{i}{a}\right] \exp [-(t-1) r]\right]$

$\frac{d F_{G}}{d r}=-a \log \left[\frac{i}{a}\right](t-1) \exp \left[-(t-1) r+\log \left[\frac{i}{a}\right] \exp [-(t-1) r]\right](24)$ 
Thus the only differences between the growth curve models are the constraints on the factor loadings in the matrix F. Figure 5 plots the factor loadings for the Gompertz curve for time points $t=1, \ldots 10$. As would be expected, the loadings on the asymptote factor increase parallel to the hei ght of the curve itself. Loadings on the initial factor are highest at the start of the process, whereas those on the rate factor are highest when growth is most rapid.

The model allows for both variation within, and covariation between the latent growth factors. So, for example, within a population, initial value might be correlated with asymptote, giving rise to one of the three possible covariances between the components of growth. Second, the model is specified for discrete, evenly spaced time periods, but it is not limited to data of this type. If we had data assessed at ages 1, 2, 4 and 7, we could create or sel ect the rows of $\mathbf{L}$ accordingly.

\section{Modeling data from relatives}

It is a relatively direct matter to extend the phenotypic growth models to cover data from relatives such as twins, but there are several important options. McArdle, ${ }^{11}$ foll lowing Vandenberg and Falkner ${ }^{24}$ presented a model of the genetic decomposition of the growth parameters. In this case, we have three covarying latent factors (asymptote, initial and rate) and uncorrel ated residual variance. When there are data from relatives, we can model the familial resemblance for both the latent factors and the residual components. A multivariate path diagram ${ }^{23}$ of such a model is shown in Figure6. In moving from Figure4, we have substituted the vector of latent variables $\mathbf{G}$ for the three growth factors $a$, $i$ and $r$, and $\mathbf{P}_{\mathrm{i}}$ represents the $\mathrm{n}_{\mathrm{t}}$ observed measures. The covari-

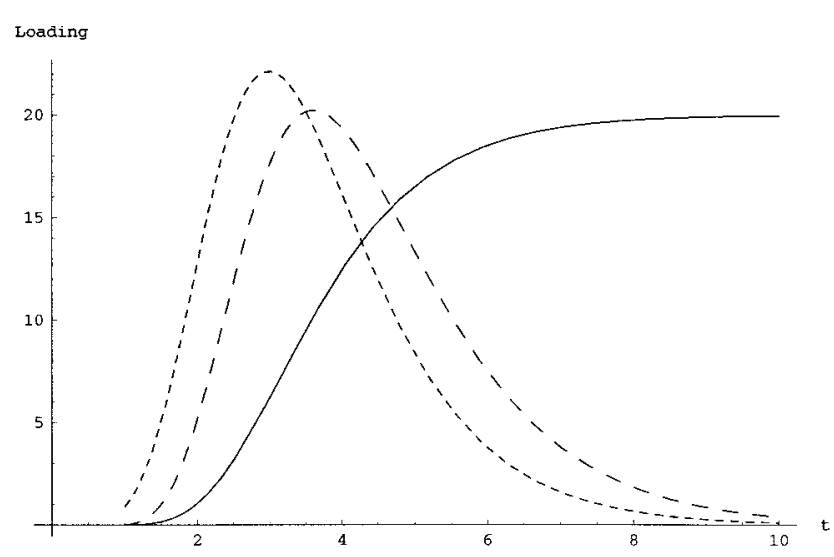

Figure 5 Plot of factor loadings according to partial derivatives of the Gompertz curve for time points $t=1, \ldots 10$. Key: - - - --asymptote, a; - - - - initial, i; - - - rate, r ance between the factors, A, I and $\mathbf{R}$ in Figure4, is now model led in the $\mathbf{A}, \mathbf{C}$ and $\mathbf{E}$ matrices. Likewise, we have substituted $\mathbf{A}_{\mathbf{s}}, \mathbf{C}_{\mathbf{s}}$ and $\mathbf{E}_{\mathrm{s}}$ for the residual variance. The model gives predicted covariance among twins which may be written:

$$
\begin{aligned}
& \Sigma=(\mathbf{I} \otimes \mathbf{F})\left(\begin{array}{cc}
\mathbf{A}^{\prime}+\mathbf{C}^{\prime}+\mathbf{E}^{\prime} & \alpha \mathbf{A}^{\prime}+\mathbf{C}^{\prime} \\
\alpha \mathbf{A}^{\prime}+\mathbf{C}^{\prime} & \mathbf{A}^{\prime}+\mathbf{C}^{\prime}+\mathbf{E}^{\prime}
\end{array}\right)(\mathbf{I} \otimes \mathbf{F})^{\prime} \\
& +\left(\begin{array}{cc}
\mathbf{A s}_{\mathbf{s}^{\prime}}+\mathbf{C}_{\mathbf{s}^{\prime}}+\mathbf{E}_{\mathbf{s}^{\prime}} & \alpha \mathbf{A}_{\mathbf{s}^{\prime}}+\mathbf{C}_{\mathbf{s}^{\prime}} \\
\alpha \mathbf{A}_{\mathbf{s}^{\prime}}+\mathbf{C}_{\mathbf{s}^{\prime}} & \mathbf{A s}_{\mathbf{s}^{\prime}}+\mathbf{C}_{\mathbf{s}^{\prime}}+\mathbf{E}_{\mathbf{s}^{\prime}}
\end{array}\right)
\end{aligned}
$$

where $\alpha=1$ for MZ and 0.5 for DZ twins, I is a $2 \times 2$ identity matrix, $\mathbf{F}$ is the factor loading matrix, and $\otimes$ denotes right kronecker product. The structure of the matrices in this model may be chosen from a variety of identified forms. All the usual tools of multivariate genetic analysis may be applied to the three covarying latent growth factors. We could, for example, use a Cholesky decomposition of the additive genetic, common and specific environmental factors if $M Z$ and $D Z$ twin data are available, in which case the matrices A, C and $\mathbf{E}$ would be lower triangular. The matrices could be restricted to represent singlefactor or independent sources of variation for the

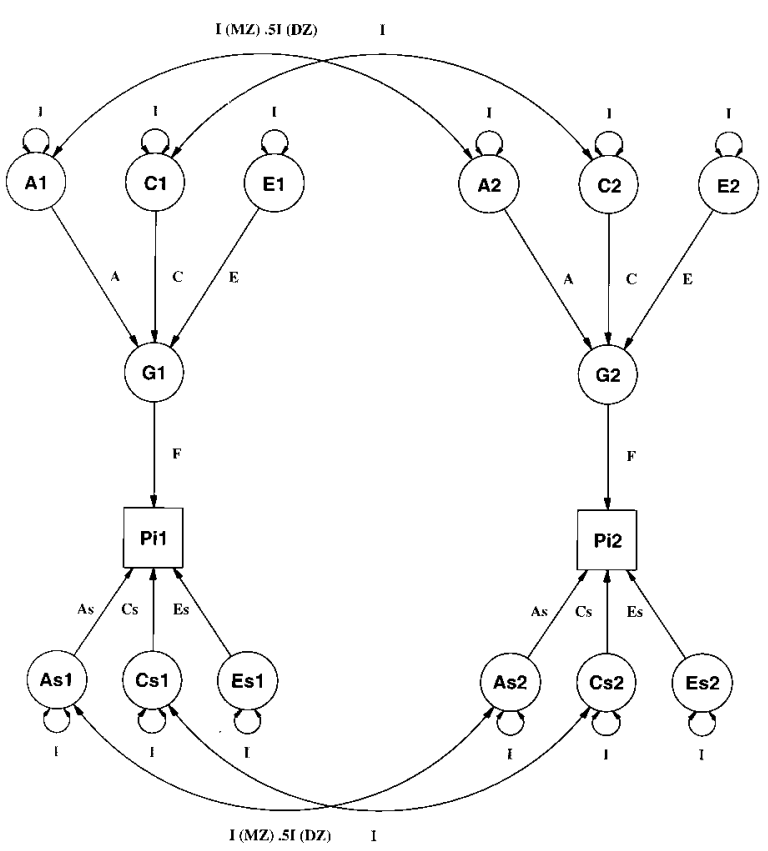

Figure 6 Multivariate path diagram of resemblance between $M Z$ or DZ twins under a growth curve model. Additive genetic, common and specific environmental components (G, C and E) act additively to produce variation and covariation in growth curve factors $G$, which in turn cause variation and covariation in the phenotypes $(\mathrm{P})$ over time. Residual components, not explained by individual differences in growth curve factors, may also be partitioned into genetic $\left(A_{s}\right)$, and environmental $\left(C_{s}\right.$ and $\left.E_{s}\right)$ components 
components by making them $3 \times 1$ or $3 \times 3$ diagonal, respectively.

We can take similar liberties with modeling the residual variation not explained by the growth curve. One approach, used here, is to allow timespecific $A, C$ and $E$ factors, which requires $\mathbf{A}_{s}, \mathbf{C}_{\mathbf{s}}$ and $\mathbf{E}_{\mathbf{s}}$ to be diagonal. In factor models, modeling of residual variation is often limited to such simple structure of the residuals because of the large number of parameters used to fit the factor structure, which can explain almost any covariance structure. However, growth curve models are highly efficient, requiring only nine parameters, regardless of the number of occasions. Many options for exploring residual covariation not explained by the growth curve are therefore avai lable, including conventional general factors or simplex models, or combinations thereof. In this article we use only the time-specific approach to modeling the residual covariances.

Note that the method described here is a singlestep analysis. This is in contrast to the approach used by Baker et $\mathrm{al}^{25}$ which uses two steps. First, individual growth curve parameters are estimated from each subject in the sample. Second, genetic and environmental parameters are estimated from the individual parameters.

\section{Application to the Bayley Infant Mental Development Scale}

Data to illustrate the approach come from the Louisville Twin Study ${ }^{26}$ and were published and analyzed by McArdle. ${ }^{11}$ The Bayley Infant Mental Devel opment scale was administered to twins aged from 6 months to 2 years, at regular 6-month intervals. As described in McArdle's paper, means, standard deviations and correl ation matrices for MZ and DZ pairs were computed from the data based on unequal sample sizes ranging from 72 to 105 . Scores were computed as 100 times raw score divided by the maximum BIMD of 163, and are thus interpreted as a 'percentage correct' metric. For analysis in this article, covariance matrices and means are used. These statistics are less than optimal for the analysis of incomplete data, especial ly since Mx is capable of analyzing raw data via maximum likelihood. ${ }^{27-30}$ However, these summary statistics are sufficient for the purposes of illustration.

An Mx script for fitting the genetic growth curve model is given in A ppendix 1 . It is relatively simple. The first group is used to compute the partial derivative vectors for each of the time points; these form the columns of matrix $\mathbf{F}$, the factor loadings. The second group sets up the $\mathbf{A}, \mathbf{C}$ and $\mathbf{E}$ components of covariance between the asymptote, initial and rate factors. The third and fourth groups fit the model to the $M Z$ and DZ data, respectively. Several submodels are then fitted using the multiple fit option.

As a starting point for comparison, we note that McArdle ${ }^{11}$ fitted a latent growth curve model with:

a) an initial level

b) unrestricted loadings on a second component; and

c) common and specific biometric components.

This model achieved a fit of $\chi^{2}=169$ on 21 degrees of freedom, compared with a saturated baseline model. We hope to improve on this fit here.

\section{Results}

Table 2 shows the parameter estimates for all three growth curve genetic models. The estimates of the means are illustrated graphically in Figure7, which also shows the estimated exponential, logistic and Gompertz growth curves, based on the parameters a, $i$ and $r$ for the four models. Evidently, the models fit the means well, as would be expected since these statistics have small standard errors relative to the covariances. The figure suggests that additional measurement occasions would help discriminate between the curves, as their closely parallel forms diverge after the two-year final measurement.

In Table2, there are $3 \times 3$ matrices reported for each of $A_{f}, C_{f}$ and $E_{f}$. Each matrix contains estimates of the $A, C$ or $E$ contribution to the covariance matrix among the three growth components: the asymptote, initial and rate. The top-left element in each matrix represents the variance in the asymptote factor, which is much greater for the $\mathrm{C}$ component than for $A$ or $E$ in the exponential model (1756.19 vs 4.76 or 4.90). The same is true, to a lesser extent, for the logistic and Gompertz models. It is al so the case that the $\mathrm{C}$ component is larger than the $\mathrm{A}$ or $\mathrm{E}$ for the variance of the initial factor (10.4 vs 1.14 or 0.16 ). There is little evidence of variation in the rate parameter; it is estimated to be near zero across all three models. This boundary condition may be one reason that the growth curve models do not fit as well as the Cholesky decomposition for these data. It suggests that the pattern of change in mean and variance does not match the partial derivative of the growth curve with respect to the rate parameter $r$.

With only four occasions, there is little information to assess individual differences in rates of growth. Likewise, there is littl e power to estimate the covariances between the additive genetic factors that contribute to variance in asymptote, initial and rate; the correl ations between these components are estimated at unity so a single factor would suffice. The same is true of the specific environmental factors in 
Table 2 Parameter estimates for three growth curve models fitted to Bayley Infant Mental Development data on MZ and DZ twins assessed on four occasions

\begin{tabular}{|c|c|c|c|c|c|c|c|c|c|c|c|c|}
\hline \multirow[b]{2}{*}{ Parameter } & \multicolumn{12}{|c|}{ Model } \\
\hline & \multicolumn{4}{|c|}{ Exponential } & \multicolumn{4}{|c|}{ Logistic } & \multicolumn{4}{|c|}{ Gompertz } \\
\hline$a$ & & & & 140.05 & & & & 97.29 & & & & 108.01 \\
\hline i & & & & 39.88 & & & & 40.17 & & & & 40.00 \\
\hline$r$ & & & & 0.17 & & & & 0.64 & & & & 0.41 \\
\hline \multirow{4}{*}{ Gf } & & $a$ & i & $r$ & & $a$ & $\mathrm{i}$ & $r$ & & $a$ & $\mathrm{i}$ & $r$ \\
\hline & a & 4.76 & -1.00 & -1.00 & a & 5.74 & 1.00 & 1.00 & $a$ & 2.88 & 1.00 & 1.00 \\
\hline & $\mathrm{i}$ & -2.32 & 1.14 & 1.00 & $\mathrm{i}$ & 2.80 & 1.37 & 1.00 & $\mathrm{i}$ & 1.89 & 1.25 & 1.00 \\
\hline & $r$ & -0.03 & 0.02 & 0.00 & $r$ & 0.02 & 0.01 & 0.00 & $r$ & 0.02 & 0.01 & 0.00 \\
\hline \multirow{4}{*}{$\mathrm{Cf}$} & & $a$ & $\mathrm{i}$ & $r$ & & $\mathrm{a}$ & $\mathrm{i}$ & $r$ & & $a$ & $\mathrm{i}$ & $r$ \\
\hline & a & 1756.19 & -0.16 & -1.00 & a & 88.66 & 0.06 & -0.90 & $a$ & 223.12 & -0.04 & -0.99 \\
\hline & $\mathrm{i}$ & -21.49 & 10.40 & 0.16 & $\mathrm{i}$ & 1.67 & 9.46 & -0.49 & $\mathrm{i}$ & -1.72 & 9.92 & -0.08 \\
\hline & $r$ & -3.07 & 0.04 & 0.01 & $r$ & -0.37 & -0.07 & 0.00 & $r$ & -0.83 & -0.01 & 0.00 \\
\hline \multirow{4}{*}{ Ef } & & $a$ & $\mathrm{i}$ & $r$ & & $\mathrm{a}$ & $\mathrm{i}$ & $r$ & & $a$ & $\mathrm{i}$ & $r$ \\
\hline & a & 146.94 & 1.00 & -1.00 & a & 8.96 & 1.00 & -1.00 & $a$ & 21.32 & 1.00 & -1.00 \\
\hline & i & 4.90 & 0.16 & -1.00 & i & 1.18 & 0.16 & -1.00 & $\mathrm{i}$ & 1.86 & 0.16 & -1.00 \\
\hline & $r$ & -0.27 & -0.01 & 0.00 & $r$ & -0.06 & -0.01 & 0.00 & $r$ & -0.10 & -0.01 & 0.00 \\
\hline & $t_{1}$ & $t_{2}$ & $t_{3}$ & $t_{4}$ & $t_{1}$ & $t_{2}$ & $t_{3}$ & $t_{4}$ & $t_{1}$ & $t_{2}$ & $t_{3}$ & $t_{4}$ \\
\hline$A_{S}$ & 2.21 & -1.19 & -2.09 & 0.00 & 2.21 & 1.19 & 2.10 & 0.00 & 2.21 & -1.18 & 2.11 & 0.00 \\
\hline $\mathrm{C}_{\mathrm{s}}$ & -1.10 & 0.00 & 0.00 & 0.00 & -1.33 & 0.00 & 0.43 & 0.00 & 1.23 & 0.00 & 0.00 & 0.00 \\
\hline $\mathrm{E}_{\mathrm{s}}$ & 2.07 & 1.96 & 1.79 & 1.94 & 2.06 & 1.95 & 1.79 & 1.94 & 2.06 & 1.96 & 1.79 & 1.94 \\
\hline
\end{tabular}

Variance in the growth curve components $a$, $i$ and $r$ due to genetic $\left(G_{f}\right)$, common environment $\left(C_{f}\right)$ and specific environment $\left(E_{f}\right)$ factors are shown on the diagonal of matrices, with covariances below and correlations above. Variance due to occasion-specific genetic and environmental factors $A_{s}, C_{s}$ and $E_{s}$ are shown for the four occasions $t_{1}$ to $t_{4}$.

growth. For shared environmental effects, which have a much greater effect on phenotypic variation, the correl ations are large between initial and rate for all models. This would suggest that shared environmental factors that contribute to initial level are very similar to those that influence rate of growth. However, variability in rate is small, and therefore these high correlations are of little consequence.

The relative contribution of the factors to the phenotypic variance changes across occasion. This is because the factor loadings are strict functions of the parameters $a, i$ and $r$ and the time point $t$. The

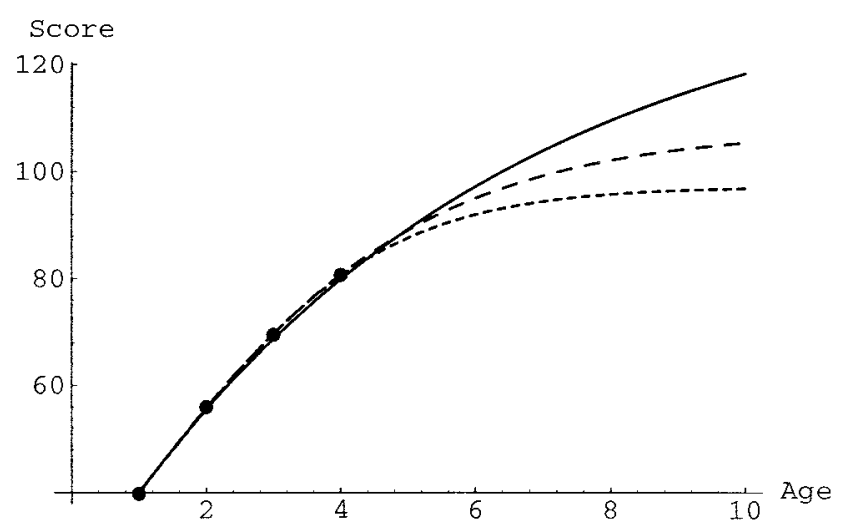

Figure 7 Plots of the exponential (- - - --), logistic (- - -) and Gompertz (- - - - ) curves with parameters estimates set to the solution of fitting the growth curve models to the MZ and DZ data on Bayley Infant Mental Development scales proportion of variance accounted for by each component could be computed for all four occasions, and for all three models. In all three models, the growth curve accounts for approximately 64\%, 88\%, 74\% and $100 \%$ of the variance in occasions one to four respectively.

Approximate $\chi^{2}$ fit statistics vary from 129 to 138 across the different growth curve models, despite their having the same number of degrees of freedom. This suggests that, even with these few occasions, information in the pattern of variances and covariances over time can effect some discrimination between the models, and that these differences would be enhanced with more occasi ons of measure ment. A further indication of model quality is the reasonableness of the parameter estimates. The logistic and Gompertz curves have asymptote parameters quite close to the theoretical maximum (100) for these test scores, whilst the exponential suggests a less plausible maximum of 139 .

The full models demonstrate the identification and feasibility of fitting genetic growth curves, and that some fit the data better than others. To test some specific hypotheses about individual differences in growth, we can compare the fit of submodels. A series of comparative fit statistics for the three growth curves is shown in Table3. On the whole, the changes in fit from a model to a submodel are similar across the three types of curve, so we focus on one curve, the logistic. The submodels address the following hypotheses: 
1. The asymptote, initial and rate factors covary;

2. Genetic factors contribute to factor variance and covariance;

3. Environmental factors contribute to factor variance and covariance; and

4. Residual variance not due to the latent growth curve does not covary between family members.

The full models do not fit very well by the $\chi^{2}$ criterion. This may be due to non-normality in the data or the use of covariance matrices as summary statistics. As we shall see below, this problem is al so present for the Cholesky model, which suggests that the loss of fit is due to inconsistencies of the four within-person covariance matrices (MZ and DZ twin 1 and twin 2). A likely consequence of this poor fit is that all $\chi^{2}$ difference statistics (the likelihood ratio tests we use to test the difference between models) are inflated (Kendall and Stuart p.230). ${ }^{31}$ Evidence for significant loss of fit would be weaker, and evidence for non-significantly poorer fit would be stronger than usual.

Table 3 Fit statistics obtained for growth curve models and submodels applied to Bayley Infant Mental Development data on $M Z$ and DZ twins

\begin{tabular}{|c|c|c|c|c|c|c|}
\hline \multirow[b]{2}{*}{ Model } & \multicolumn{3}{|c|}{ Fit statistic } & \multicolumn{3}{|c|}{ Difference $\chi^{2}$} \\
\hline & $\chi^{2}$ & d.f. & AIC & $\chi^{2}$ & d.f. & $P$ \\
\hline \multicolumn{7}{|l|}{ Exponential } \\
\hline Full & 129.06 & 55 & 19.06 & - & - & - \\
\hline Orthogonal & 143.20 & 64 & 15.20 & 14.14 & 9 & 0.12 \\
\hline No $A$ & 140.78 & 61 & 18.78 & 11.72 & 6 & 0.07 \\
\hline No C & 169.27 & 61 & 47.27 & 40.21 & 6 & 0.00 \\
\hline No E & 134.15 & 61 & 12.15 & 5.09 & 6 & 0.53 \\
\hline No $A_{s}, C_{s}$ & 166.86 & 63 & 40.86 & 37.80 & 8 & 0.00 \\
\hline $\mathrm{No} \mathrm{A}_{\mathrm{s}}$ & 136.88 & 59 & 18.89 & 7.82 & 4 & 0.10 \\
\hline $\mathrm{No} \mathrm{C}_{s}$ & 129.21 & 59 & 11.21 & 0.15 & 4 & 0.95 \\
\hline \multicolumn{7}{|l|}{ Logistic } \\
\hline & 137.89 & 55 & 27.89 & - & - & - \\
\hline Orthogonal & 148.73 & 64 & 20.73 & 10.84 & 9 & 0.29 \\
\hline No A & 149.49 & 61 & 27.49 & 11.60 & 6 & 0.07 \\
\hline No $C$ & 177.04 & 61 & 55.04 & 39.15 & 6 & 0.00 \\
\hline No E & 142.74 & 61 & 20.74 & 4.85 & 6 & 0.56 \\
\hline $\mathrm{No} \mathrm{A}_{s}, \mathrm{C}_{\mathrm{s}}$ & 183.91 & 63 & 57.91 & 46.02 & 8 & 0.00 \\
\hline $\mathrm{No} \mathrm{A}_{s}$ & 145.83 & 59 & 27.83 & 7.94 & 4 & 0.05 \\
\hline $\mathrm{NoC}_{\mathrm{s}}$ & 138.30 & 59 & 20.30 & 0.41 & 4 & 0.98 \\
\hline \multicolumn{7}{|l|}{ Gompertz } \\
\hline Full & 31.93 & 55 & 21.93 & - & - & - \\
\hline Orthog & 144.13 & 64 & 16.13 & 12.20 & 9 & 0.20 \\
\hline No A & 143.54 & 61 & 21.54 & 11.60 & 6 & 0.07 \\
\hline No & 171.72 & 61 & 49.72 & 39.79 & 6 & 0.00 \\
\hline No & 137.00 & 61 & 15.00 & 5.07 & 6 & 0.5 \\
\hline No $A_{s}, C_{s}$ & 172.76 & 63 & 46.76 & 40.83 & 8 & 0.00 \\
\hline $\mathrm{No}_{\mathrm{s}}$ & 139.81 & 59 & 21.81 & 7.88 & 4 & 0.01 \\
\hline $\mathrm{NoC}_{\mathrm{s}}$ & 132.21 & 59 & 14.21 & 0.27 & 4 & 0.99 \\
\hline \multicolumn{7}{|c|}{ Cholesky and Simplex } \\
\hline Full Cholesk & 117.75 & 50 & 17.75 & - & - & - \\
\hline & & 6 & 8.59 & - & - & - \\
\hline Simplex & 135.64 & 65 & 5.64 & - & - & - \\
\hline
\end{tabular}

The orthogonal model does not fit significantly worse than the full model, so there is no evidence that the growth curve components covary. There is little evidence for either genetic or random environmental variation in the growth curve components, but significant variation is associated with the shared environment. The picture for the residual variance is quite different; familial resemblance appears to be additive genetic rather than shared environmental in origin. Thus of our four hypotheses above, 1, 2 and 4 are rejected and 3 is not.

\section{Comparison with Cholesky and Simplex}

To provide a comparative framework for the fit of the growth curve models, we fitted two standard behavior genetic models for longitudinal data, the Cholesky and the Simplex. The A, C, E Cholesky (Figurela) gave a fit of 117.75 , with $50 \mathrm{df}$ which yields Akaike's Information Criterion, $\mathrm{AIC}^{32}$ $=17.75$. Several of the growth curve submodels fitted better by AIC, so the poor fit (significant $\chi^{2}$ ) to these data does not seem to be a feature of growth curves models per se.

We followed Dolan's ${ }^{7}$ treatment and specified separate A, C and E factor means which were constrained to equal a constant times the variance of each factor. The simplex model has a lower AIC (is more parsimonious) than most of the growth curves $\chi^{2}=134.59, \mathrm{df}=63, \mathrm{AIC}=8.59$ ). It allows for $\mathrm{A}, \mathrm{C}$ and $E$ transmission and innovation components as shown in Figure 1b. Further improvement in AIC was found by el iminating the means on the common and random environment components $\left(\chi^{2}=135.64\right.$, $\mathrm{df}=65, \mathrm{AIC}=4.64$ ), as they had only minor effects on the goodness of fit $\chi^{2}$. Though this 'genetic means' model fits well, there seems no theoretical justification to expect that genetic factors affect the means whereas environmental factors - especially shared environmental which are not due to measurement error - do not.

\section{Discussion}

We have reviewed growth curve theory from a dynamical systems perspective. In addition to its didactic value, it opens the door to genetic modeling of more complex dynamical systems within the structural equation modeling framework. Both balanced and incomplete data can be anal yzed with this method.

The illustrative application to the published BIMD data has some novel substantive conclusions. The variance growth curve parameters are largely under shared environmental control is in agreement with McArdle's ${ }^{11}$ results for linear latent growth models. 
We have expanded on this initial treatment both by specifying different functional forms for growth (logistic etc.) and by partitioning the residual variance into $A, C$ and $E$ components. This latter partition is quite informative; in all model s we found evidence for genetic factors in the residual variance, which suggests several possi bilities. First, we may be measuring cognitive development at too few and too widel y spaced intervals of time. Perhaps what we are ascribing to occasion-specific variance encompasses other growth processes which are partly under genetic control. Alternatively, it might be that the response of the individual to testing on that particular occasion (the 'person $\times$ situation interaction') is genetically mediated. It seems difficult to discriminate between these possibilities without additional data.

Inferences we draw from the application should be viewed in the light of several potentially important limitations. First, the BIMD scores were treated as raw percentage scores, and this may not be appropriate for a scale that was not developed with, for example, Rasch ${ }^{33}$ scaling. $^{34,35}$ Second, we would expect similar answers if the raw data were anal yzed instead of the means and covariances used here, but differences might occur if the data are not missing completely at random. It would be interesting to see how robust the findings are with raw data. Third, we have ignored the effect of sex in these analyses. Substantial sex differences in growth parameters might have inflated our estimates of common environmental variance. Future analyses separating males and femal es would be valuable, al though quite large sample sizes would be required to detect sex differences in the model parameters.

Possible extensi ons to the methods presented here are many. First, it would be very simple to apply them to data collected from larger pedi grees than the pairs used here. Such datasets could include irregular family structures, with varying ages. A practical limitation of such studies - especially of cognitive development - would be finding parents who had been assessed in their early childhood. However, with appropriately scaled tests it should be possible to anal yse data across wi de age ranges. It may not be necessary to assess parents and their children at comparable ages, so long as there is sufficient overlap between the two samples. Indeed nonoverl apping samples might suffice if, as in this study, the sources of variability appear to be entirely familial in origin, but we would advocate against designing studies which cannot assess the lasting impact of specific environmental factors. Measurement of different-aged relatives would be a poor substitute for longitudinal data, whose cost to collect can be reduced with cohort-sequential designs. ${ }^{36}$ While parents and offspring may be the most obvious addition to a twin study, other collateral relative groups - such as husbands and wives or full and half-siblings may be used in the short term to test assumptions of the twin study.

Second, the methods could be combined with those of standard behavior genetic analysis to enable testing of many types of hypothesis. For example, we could test for sibling interaction in development by modeling differences in $\mathrm{MZ}$ and $\mathrm{DZ}$ variance in the growth factors. We might test for $\mathrm{G} \times \mathrm{E}$ interaction by testing for heterogeneity of the growth curve parameters - or even types of growth curve between groups subdivided according to some environmental factor. Multivariate extensions are readily incorporated, where we could allow for growth factors which influence several different variables simultaneously, or feedback mechanisms between the growth factors of different variables. Similarly, quantitative trait loci for the growth factors could be sought with appropriate data on DNA markers. ${ }^{37,38}$ Future work on these methods has great potential for understanding individual differences in change which could be applied to many areas of human development.

Extensions to ordinal or binary data require additional considerations. We could impose thresholds at various heights on the curve and via numerical integration compute the predicted proportion that would lie between each pair of consecutive thresholds. However, for two time points and twins we have four variables, requiring four-dimensional integration to compute the joint likelihood. While this is feasible with current computer hardware and software, studies with more than six occasions of measurement per subject (or 12 per family when larger pedigrees are considered) would prove difficult to analyze. Polychoric correlations and their associated weight matrices (ADF methods ${ }^{39}$ ) can be a useful way to summarize ordinal data, and they would capture changes in covariance over time. However, they would lack the information on changes in mean and variance which provide much of the information to fit growth curves. Perhaps joint analysis of polychorics and thresholds and their overall weight matrix would be a good approach, although the large sample sizes required for ADF methods are presumably even larger when the thresholds are included.

Finally, we recognize the limitations to dynamical systems; many differential equations cannot be solved, and that even those that can may not be identified when implemented as a structural model. For example, the general sine wave function a $\sin [b+c t]$ has partial derivatives which are linearly dependent, so the three factor model is not identified. Nevertheless, the methods offer much promise for modeling change over time. While time is the 
natural dimension with which to study change, other dimensi ons such as physical distance might be substituted to study genetic and environmental variation in other phenomena such as EEG waves or cellular chemical concentrations. Future research on these methods could grow in many directions.

\section{Acknowledgements}

This research was supported by $\mathrm{NIH}$ grants RR-08123, MH/AA-49492, AA-09095 and MH-01458 to the first author, and NIA grant AG-07137 to the second.

\section{References}

1 Browne MW. Structured latent curve models. In: Cuadras CM and Rao CR (eds.), Multivariate Analysis: Future Directions. Elsevier Science: Amsterdam, 1993, pp 171-197.

2 Browne MW, Du Toit SHC. Models for learning data. In: Collins LM and Horn JL (eds.), Best Methods for the Analysis of Change, American Psychological Association: Washington DC, 1991; pp 47-68.

3 Neale MC, Cardon LR. Methodology for Genetic Studies of Twins and Families. Dordrecht, NL: Kluwer Academic Publishers: 1992.

4 Horn JL, Little KB. Methods for isolating change and invariance in patterns of behavior. Multivariate Behav Res 1966; 1: 219-229.

5 Thurstone LL. Multiple Factor Analysis. University of Chicago Press: Chicago, 1947.

6 Dolan C. PhD thesis, Biometric Decomposition of Phenotypic Means in Human Samples, University of Amsterdam, 1992.

7 Dolan CV, Molenaar PCM, Boomsma DI. Longitudinal genetic analysis of Iongitudinal means and covariance structure in the simplex model using LISREL. Behav Genet 1991; 21: 49-61.

8 Hewitt JK, Eaves LJ, Neale MC, Meyer JM. Resolving causes of developmental continuity or 'tracking'. I. Longitudinal twin studies during growth. Behav Genet 1988; 18: 133-151.

9 Braun M. Differential Equations and their Applications. Springer Verlag: New York, 1991.

10 Vandenberg SG, Falkner F. Hereditary factors in human growth. Hum Biol 1965; 37: 357-365.

11 McArdle JJ. Latent variable growth within behavior genetic models. Behav Genet 1986; 16: 163-200.

12 McArdle JJ, Goldsmith HH. Alternative common-factor models for multivariate biometric analyses. Behav Genet 1990; 20: 569-608.

13 Murray JD. Mathematical Biology. Springer Verlag: Berlin, 1989.

14 Wolfram S. Mathematica. Addison-Wesley: Reading, MA, 1988.

15 Verhulst PF. Notice sur la loi que la population suit dans son accroissement. Corr Math Phys 1838; 10: 113-121.

16 Gompertz B. Philosophical Transactions, 1825.

17 Martin R, Teo KL. Optimal Control of Drug Administration in Cancer Therapy. World Scientific: New Jersey, 1994.

18 Juckett DA, Rosenberg B. Comparison of the Gompertz and Weibull functions as descriptors for human mortal ity distributions and their intersections. Mech Ageing Dev 1993; 69: $1-31$

19 Wright S. The method of path coefficients. Ann Math Stat 1934; 5: 161-215.
20 Loehlin JC. Latent Variable Models. Lawrence Erlbaum: Baltimore, 1987

21 Everitt BS. An Introduction to Latent Variable Models. Chapman and Hall: London, 1984.

22 McArdle JJ, Boker SM. RAMpath path diagram software. Data Transforms Inc: Denver, CO, 1990.

23 Vogler GP. Multivariate path analysis of familial resemblance. Gene Epidemiol 1985; 2: 35-53.

24 Vandenberg SG. Multivariate analysis of twin differences. In: Vandenberg SG (ed.) Methods and Goals in Human Behavior Genetics Academic Press: New York, 1965, pp 29-43.

25 Baker LA, Reynolds C, Phelps E. Biometrical analysis of individual growth curves. Behav Genet 1992; 22: 253-264.

26 Wilson RS. The Louisville Twin Study: Developmental synchronies in behavior. Child Dev 1983; 54: 298-316.

27 Neale MC, Boker SM, Xie G, Maes HH. Mx: Statistical Modeling, 5th edn. MCV, Richmond, VA, 1999.

28 McArdle JJ. Structural factor analysis experiments with incomplete data. Multivariate Behav Res 1994; 29: 409-454.

29 McArdle JJ, Allison DB. Regression change models with incomplete repeated measures data in obesity research. In: Allison DB and Pi-Sunyer FX (eds.). Obesity Treatment, Plenum Press: New York, 1995, pp 53-63.

30 McArdle JJ, Hamagami F. Multilevel models from a multiple group structural equation perspective. In: Marcoulides G, Schumacker E (eds.). Advanced Structural Equation Modeling. Lawrence Erlbaum: Hillsdale, NJ, 1996, pp 89-124.

31 Kendall MG, Stuart A. The Advanced Theory of Statistics. Vol. 2: Inference and Relationship. Hafner: New York, 1961.

32 Akaike H. Factor analysis and AIC. Psychometrika 1987; 52: 317-332.

33 Rasch G. On the meaning of measurement in psychology. In: Neyman J (ed.), Proceedings of the 4th Berkel ey Symposium on Mathematical Statistics and Probability, University of California Press: Berkeley, 1961.

34 Embretson SE. Implications of multidimensional latent trait model for measuring change. In: Collins LM, Horn JL (eds.). Best Methods for the Analysis of Change. American Psychological Association: Washington, DC, 1991; pp 184-197.

35 Woodcock RW. Theoretical foundations of the WJ-R measures of cognitive ability. Conference on intelligence: Theories and practice (1990, Memphis, Tennessee). J Psychoed Assess 1990; 8(3): 231-258

36 Bell RQ. Convergence: An accelerated Iongitudinal approach. Child Dev 1953; 24: 145-152.

37 Eaves LJ, Neale MC, Maes HH. Multivariate multipoint linkage analysis of quantitative trait loci. Behav Genet 1996; 26: 519-526.

38 Neale MC. QTL mapping with sib-pairs: The flexibility of Mx. Greenwich Medical Media: London, 1999.

39 Browne MW. Asymptotically distribution-free methods for the analysis of covariance structures. Br J Math Stat Psychol 1984; 37: 62-83.

\section{Appendix 1 Mx script to fit logistic growth curve to twin data}

\section{!}

! Mx script to fit logistic growth curve to data collected from

! twins on nocc occasions of measurement !

\#define nt 4 ! Number of time points

\#define nt $\times 8$ ! Twice number of time points 
Group 1: compute factor loading vectors ! Title of group 1

Calculation Ngroups $=5$ ! Type of group \& total No. of groups

Begin Matrices; ! declare matrices

A full 11 free ! asymptote parameter

I full 11 free ! initial parameter

$\mathrm{R}$ full 11 free ! rate parameter

T full nt 1 ! vector of occasions $t=1, \ldots$ nt

U unit nt 1

End Matrices

! A few starting values

Matrix T12345678910! extend if nt $>10$

Start 100 a 11

Start 10 i 11

Start 1 r 11

! Boundaries for the parameters

Bound -500500 all

!

! Now we use matrix algebra to compute the vectors and paste them together

! into a single matrix of factor loadings, $F$ !

Begin Algebra;

$\mathrm{J}=\mathrm{i} @+(\mathrm{a}-\mathrm{i}) @(\exp (-(\mathrm{t}-\mathrm{u}) @ \mathrm{r})) ;$ ! Logistic function itself

$\mathrm{K}=(\mathrm{i} @ \mathrm{u}-(\backslash \exp (-(\mathrm{t}-\mathrm{u}) @ \mathrm{r})) .(((\mathrm{a} * \mathrm{i}) @ \mathrm{U}) \% \mathrm{~J})) \quad \% \quad \mathrm{~J} ;$ !dj/da

$\mathrm{L}=((\mathrm{a} @ \mathrm{u})$ - (u-(( exp(-(t-u)@r))). (((a*i)@)\%J) ) \%

$\mathrm{J} ; ! \mathrm{dJ} / \mathrm{di}$

$M=(((a-i) @(t-u)) .(\backslash \exp (-(t-u) @ r)) .(((a * i) @) \% J))$

$\% \mathrm{~J} ; ! \mathrm{dJ} / \mathrm{dr}$

$\mathrm{F}=\mathrm{K}|\mathrm{L}| \mathrm{M}$;

End Algebra;

End Group

Group 2 Compute the MZ and DZ twin pairs' predicted factor covariances

Calculation

Begin Matrices

$\mathrm{H}$ full 11 ! to put 0.5 in for $\mathrm{DZ}$ twin genetic covariance

$X$ Lower 33 free ! lower triangular decomposition used initially

Y Lower 33 free

Z Lower 33 free

End Matrices;

Matrix $\mathrm{H} .5$

Start $2 \times 11 Y 11 Z 11$

Start $0.1 \times 22 \times 33$ Y 22 Y $33 Z 22 Z 33$

Begin Algebra;

$\mathrm{A}=\mathrm{X}^{*} \mathrm{X}^{\prime}$;

$C=Y^{*} Y^{\prime}$;

$\mathrm{E}=\mathrm{Z}^{*} \mathrm{Z}^{\prime}$;

$\mathrm{M}=\mathrm{A}+\mathrm{C}+\mathrm{E} \mid \mathrm{A}+\mathrm{C}-$

$A+C \mid A+C+E$;

$\mathrm{D}=\mathrm{A}+\mathrm{C}+\mathrm{E} \mid \mathrm{h} @ \mathrm{~A}+\mathrm{C}-$

$h @ A+C \mid A+C+E /$
End Algebra;

End Group

Group 3 fit the model to the MZ data

Data Ninput $=\mathrm{nt} \times 2$ nobs $=75$

CMatrix full file = bayleymz.cov ! observed cov matrix

Mean file $=$ bayleymz.mean ! observed means

Matrices

F Computed $=\mathrm{F} 1$ ! Factor loading matrix

R Computed $=$ M2 ! MZ factor covariances

A diag nt nt free ! Residual genetic factors (occasion specific)

C diag nt nt free ! Residual C factors

$\mathrm{E}$ diag nt nt free ! Residual $\mathrm{E}$ factors

I iden 22

$\mathrm{U}$ unit 12

$\checkmark$ unit 22

$X$ full 31

End Matrices

Specify X 120

Start $3 \mathrm{E} 11$ to E 44

Bound $0.520 \mathrm{E} 11$ to E 44

Means U@ $(F * X)^{\prime}$ /

Covariance (I@F) \& $R+I @\left(E^{*} E^{\prime}\right)+V @(C-$

$\left.*^{*} C^{\prime}+A^{*} A^{\prime}\right) /$

Option rs

End Group

Group 4 fit the model to the DZ data

Data ninput $=\mathrm{nt} \times 2$ nobs $=75$

CMatrix full file = bayleydz.cov

Means file = bayleydz. mean

Begin Matrices;

F Computed = F1 ! Factor loading matrix

R Computed + D2 ! DZ factor covariances

A diag nt nt $=A 3$ ! Residual genetic factors (occasion specific)

$\mathrm{C}$ diag nt nt $=\mathrm{C} 3$ ! Residual $\mathrm{C}$ factors

$\mathrm{E}$ diag nt $\mathrm{nt}=\mathrm{E} 3$ ! Residual $\mathrm{E}$ factors

H Full $11=\mathrm{H} 2$

I Iden 22

$U$ unit 12

$\mathrm{V}$ unit 22

$X$ full $31=X 3$

End Matrices;

Means U@(F*X)' /

Covariance $(I @ F) \& R+I @\left(E^{*} E^{\prime}\right)+V @\left(C^{*} C^{\prime}\right)+(H-$ $@(V+I)) @\left(A^{*} A^{\prime}\right) /$

Option rs ! Print residuals

Option Multiple ! Fit submodels next

End Group !

! Save current solution then fit seven submodels from above solution

!

save baylog.mxs

! 
! 1. make asymptote, initial and rate factors orthogonal

! drop 171920

drop 578

drop 111314

end

! 2. delete all genetic effects on factors

get baylog.mxs

drop 456789

end

! 3. delete all $\mathrm{C}$ effects on factors

get baylog.mxs

drop 101112131415

end

! 4. delete all E effects on factors get baylog.mxs

drop 161718192021

end

! 5. delete $\mathrm{G}$ parts of residuals

get baylog.mXs

drop 22232425

end

! 6. delete $\mathrm{C}$ parts of residuals

get baylog.mxs

drop 26272829

end

! 7. delete $\mathrm{G} \& \mathrm{C}$ parts of residuals

get baylog.mxs

drop 2223242526272829

end 